\title{
(6) OPEN ACCESS \\ Simulation-based training and assessment of non-technical skills in the Norwegian Helicopter Emergency Medical Services: a cross-sectional survey
}

\author{
Håkon B Abrahamsen, ${ }^{1,2,3,4}$ Stephen J M Sollid, ${ }^{1,5,6}$ Lennart S Öhlund, ${ }^{7}$ \\ Jo Røislien, ${ }^{5,8}$ Gunnar Tschudi Bondevik ${ }^{2,3}$
}

\begin{abstract}
- Additional material is published online only. To view please visit the journal online (http://dx.doi.org/10.1136/ emermed-2014-203962).

For numbered affiliations see end of article.
\end{abstract}

\section{Correspondence to} Håkon Bjorheim Abrahamsen, Department of Research and Development, Norwegian Air Ambulance Foundation, P0 Box 94, Drøbak 1441, Norway;

hakon.bjorheim.abrahamsen@ norskluftambulanse.no

Received 4 May 2014 Revised 14 September 2014 Accepted 9 October 2014 Published Online First 24 October 2014

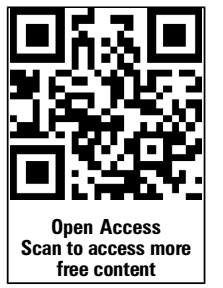

\section{ABSTRACT}

Background Human error and deficient non-technical skills (NTSS) among providers of ALS in helicopter emergency medical services (HEMS) is a threat to patient and operational safety. Skills can be improved through simulation-based training and assessment.

Objective To document the current level of simulationbased training and assessment of seven generic NTSs in crew members in the Norwegian HEMS.

Methods A cross-sectional survey, either electronic or paper-based, of all 207 physicians, HEMS crew members (HCMs) and pilots working in the civilian Norwegian HEMS (11 bases), between 8 May and 25 July 2012.

Results The response rate was $82 \%(n=193)$. A large proportion of each of the professional groups lacked simulation-based training and assessment of their NTSs. Compared with pilots and HCMs, physicians undergo statistically significantly less frequent simulation-based training and assessment of their NTSs. Fifty out of 82 (61\%) physicians were on call for more than 72 consecutive hours on a regular basis. Of these, $79 \%$ did not have any training in coping with fatigue. In contrast, 72 out of $73(99 \%)$ pilots and HCMs were on call for more than 3 days in a row. Of these, $54 \%$ did not have any training in coping with fatigue.

Conclusions Our study indicates a lack of simulationbased training and assessment. Pilots and HCMs train and are assessed more frequently than physicians. All professional groups are on call for extended hours, but receive limited training in how to cope with fatigue.

\section{INTRODUCTION}

In Norway, physician-manned air ambulance helicopters support ground ambulances in emergency missions for care and retrieval, and provide interhospital transfer of patients. The provision of ALS to critically ill and injured patients in helicopter emergency medical services (HEMS) is a complex process characterised by shifting workload and goals, ill-structured problems, uncertainty, intense time pressure, high stakes and a set of individually complex and interacting tasks of flight-operative, medical, technical, rescue and multidisciplinary character. ${ }^{1}$ This process is prone to human error, adverse events and ultimately iatrogenic injury, ${ }^{2}{ }^{3}$ which are to a large degree preventable. ${ }^{145}$ The Norwegian HEMS conduct more than 7500 urgent and interhospital air medical patient transfers annually. More than $60 \%$ of these patients are critically ill or injured (National Advisory Committee for Aeronautics (NACA) score 4-6), and more than $12 \%$ are mechanically ventilated. ${ }^{6}$

\section{Key messages}

What is already known on this subject?

- Human error and deficient non-technical skills among providers of ALS in helicopter emergency medical services (HEMS) is a threat to patient and operational safety.

- Skills can be improved through simulationbased training and assessment.

- Crew resource management is a safety management strategy, mandatory for crew members in HEMS, intended to train and assess non-technical skills.

What might this study add?

- A significant number of crew members in the Norwegian HEMS lacked simulation-based training in, and assessment of, generic nontechnical skills.

- All professional groups in HEMS are on call for extended hours but receive limited training in how to cope with fatigue.

Major adverse events in HEMS are rare, but the overall incidence of adverse events remains unknown. ${ }^{2}$ Poor interdisciplinary communication seems to be a significant factor in adverse events in air ambulance services ${ }^{7}$ and during trauma resuscitation. ${ }^{8}$ Baseline haemodynamic instability, mechanical ventilation and on-scene calls are factors associated with increased risk of life-threatening events in transit. ${ }^{9}$ Human error in any of these settings can be fatal.

Crew resource management (CRM) is a conglomerate of multidisciplinary, safety-management principles and training interventions designed to reduce human error by enhancing non-technical skills (NTSs). ${ }^{10}{ }^{11}$ NTSs can be defined as 'the cognitive, social and personal resource skills that complement technical skills, and contribute to safe and effective task performance'. ${ }^{12}$ Seven generic categories of NTSs have been suggested: situation awareness, decision-making, communication, teamwork, leadership, managing stress and coping with fatigue. ${ }^{12}$ Systematic training and assessment of NTSs in HEMS has received little attention in the past, although CRM training is required for all crew members. The time-pressured HEMS environment is not particularly suited for experiential training of NTSs. 
Simulation-based training and assessment of NTSs, as one of several CRM training interventions, is called for and recommended. ${ }^{13-16}$ Multiprofessional simulation allows repetitive practice in rare conditions and potentially dangerous operations in a safe environment, reinforces understanding across disciplines, and permits real teams to train based on the knowledge of challenges and deficiencies. ${ }^{15}{ }^{16}$ Simulation-based trauma team training has shown a significant effect on learning and team performance. ${ }^{15-17}$ Simulation is a useful tool for developing NTSs. ${ }^{14}$

The aim of this study was to document the current level of simulation-based training and assessment of a generic set of basic NTSs among crew members of the Norwegian HEMS. We hypothesised that crew members lacked simulation-based training in, and assessment of, NTSs. We also hypothesised that the extent of simulation-based training and assessment of these skills differed across the professional groups in this service.

\section{METHODS}

\section{Setting}

Eleven civilian HEMS bases operate in Norway today. Work is carried out by a small team (crew). Three crew members is the main crew concept. Each individual belongs to a separate profession. All of these professionals have their own group cultures and team dynamics, with different backgrounds and expertise, and they often work together only for a short period of time. The individual with his/her professional background is the basic building block from which HEMS crews are formed. ${ }^{12}$ In addition, team composition is continually shifting. This is why we chose to stratify our analysis by profession.

The pilot is the mission commander and has primary responsibility for flight safety and navigation; the HEMS crew member (HCM) is responsible for rescue operations and assists the physician on-scene and the pilot during flight operations; meanwhile, the physician is a certified or in-training anaesthesiologist responsible for patient treatment and care on-scene and during transportation to the hospital. Only one base operates with a nurse on board in addition to the aforementioned three-man crew. This is a local adaptation and is thus not representative of the general crew composition.

\section{Questionnaire}

Eight question categories relating to education and training in NTSs were presented as an extension of a patient safety climate questionnaire (see online supplementary appendix, section I). The present study focuses on the two question categories documenting the overall extent of simulation-based training (question I6) and assessment (question I7) on a four-point ordinal scale $(0,1-2,3-5,>5$ times per year). Both question categories contained seven questions, one for each of the aforementioned seven generic NTS categories: (1) decision-making, (2) leadership, (3) communication, (4) situation awareness, (5) teamwork, (6) managing stress and (7) coping with fatigue. ${ }^{12}$

The questionnaire contained information on one possible explanatory variable: the maximum number of consecutive on-call duty hours, reported on a seven-point ordinal scale.

\section{Data collection}

Between 8 May and 25 July 2012, we conducted an anonymous, cross-sectional survey among all 207 physicians, HCMs and pilots working in the civilian Norwegian HEMS. To maximise the response rate, a commentary on the upcoming study was published in the Norwegian Medical Journal. ${ }^{18}$ The survey was distributed via both e-mail, with a link to a web-based questionnaire (Questback), and an identical paper version (see online supplementary appendix) along with prepaid stamped return envelopes. After 2-4 weeks, all crew members received a follow-up phone call as a reminder and encouragement to answer.

Questionnaires returned with missing data on occupation or profession were excluded. We also excluded those with more than $50 \%$ missing values in order to maintain consistency with an upcoming psychometric analysis from other parts of the questionnaire relating to safety climate, but not within the scope of this survey (see online supplementary appendix). Respondents were excluded if they did not work in the civilian HEMS (eg, military search and rescue helicopter or aeroplane) and if they did not belong to the aforementioned target group of professionals (eg, nurses and paramedics).

\section{Statistical analysis}

Our unit of analysis is the professional groups rather than the HEMS crew as a whole. Descriptive data are presented as ratios or numbers. Spearman's correlation $\left(r_{s}\right)$ was calculated to assess the inter-item association between each of the seven items in question categories I6 and I7. Frequency of simulation-based training and assessment of NTSs across all professional groups is presented as bar charts. The group of nurses was considered too small $(n=6)$ to allow comparison of professional groups in a rigorous statistical analysis.

To assess possible differences in simulation-based training and assessment between professions, we dichotomised the items $(0=$ no training/assessment, $1=$ some training/assessment $)$ and used them as dependent variables in a series of logistic regression models, with crew type as a three-level nominal explanatory variable: physician, HCM and pilot. The last of these was used as the reference group, since the aviation industry has led the field and driven formal assessment of individual pilot's NTSs. ${ }^{19}$ Results are presented as OR with 95\% CI.

Fisher's exact test was used to explore the association between crew members working for the health enterprise (physicians) or the flight operators (HCMs and pilots) and three dichotomised variables by using a two-by-two design: on-call duty hours $(0=$ less than or equal to $72 \mathrm{~h}, 1=$ more than $72 \mathrm{~h})$; simulation-based training and assessment $(0=$ no training/assessment, $1=$ some training/assessment). Results are presented as ratios (\%) and numbers, and a $\mathrm{p}$ value less than 0.05 was considered significant.

SPSS V.18.0 and the freeware R 2.12 were used for all calculations.

\section{Ethics}

This study was conducted in compliance with the ethics guidelines of the Helsinki Declaration. All participants received written information about the purpose of the study, and were told that the data would be collected anonymously and treated in confidence. The regional ethics committee of South-Eastern Norway (reference number 2010/3326) and the Norwegian Social Science Data Services reviewed and approved the study. Written informed consent was considered unnecessary, since responding to the questionnaire was voluntary.

\section{RESULTS}

Of the 207 people working at the 11 Norwegian HEMS bases, 172 responded (150 electronically, 22 on paper via mail), of which 158 were eligible for inclusion. Accordingly, the response rate was $81.8 \%$ (figure 1). All HEMS bases were represented among the respondents. Of the included respondents, 82 


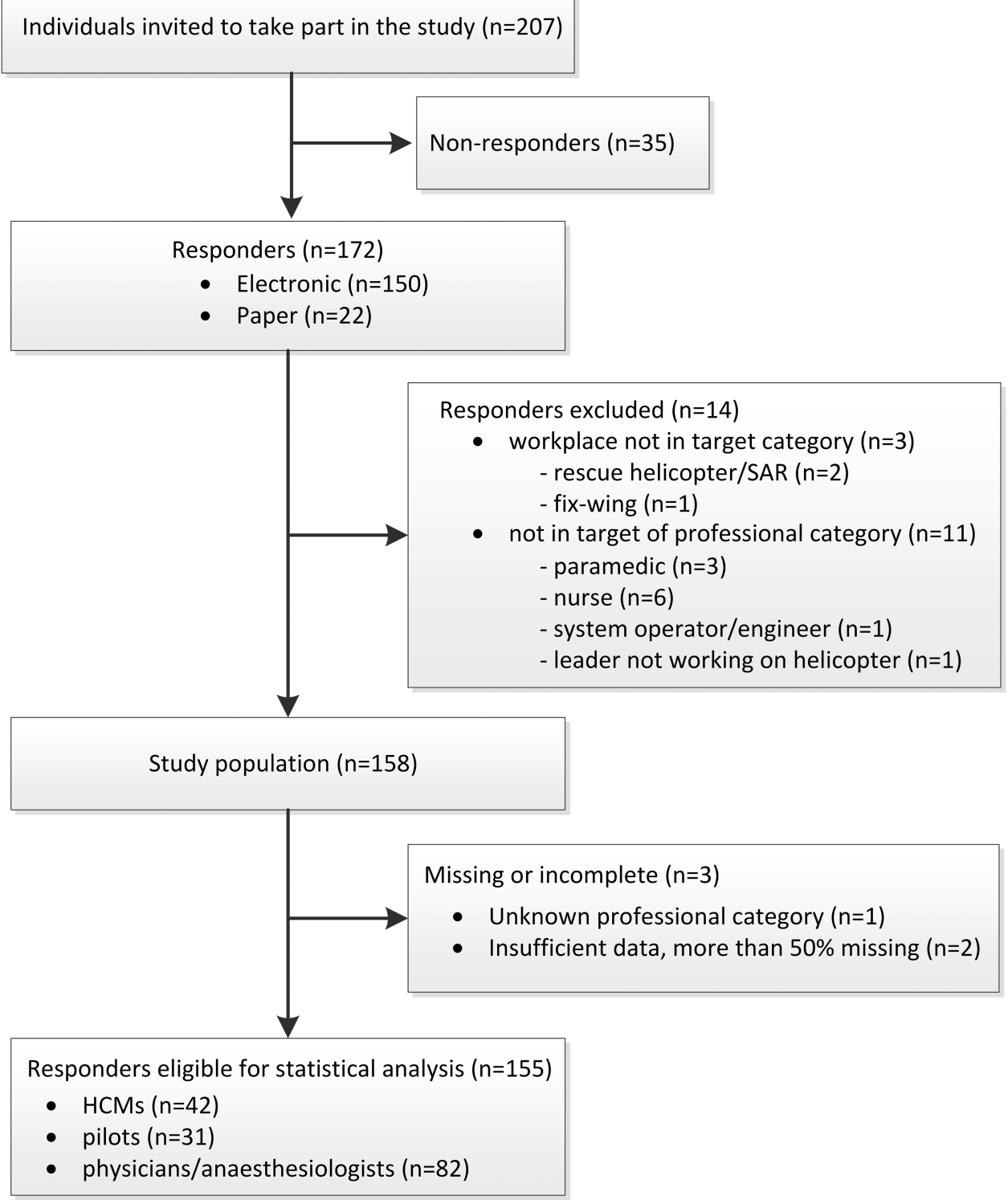

Figure 1 Participant flow through the study illustrating inclusion/exclusion of respondents. HEMS, helicopter emergency medical service; HCM, HEMS crew member; SAR, search and rescue.

(52.9\%) were working for the health enterprise, and 73 (47.1\%) for the flight operator. None of the HCMs had less than 5 years of prehospital experience. In contrast, 26 of the 82 physicians $(31.7 \%)$ and 13 of the 32 pilots had less than 5 years of prehospital experience. Of the HCMs, 33 (78.6\%) had more than 10 years of prehospital experience.

There is a strong correlation $\left(0.68 \leq \mathrm{r}_{\mathrm{s}} \leq 0.89\right)$ between the generic NTS categories 1-6 related to the simulation-based training of NTSs (table 1, question category I6). Correlation between these six categories and the skill category 'coping with fatigue' was generally somewhat smaller $\left(0.53 \leq \mathrm{r}_{\mathrm{s}} \leq 0.78\right)$. There is also a strong correlation $\left(0.77 \leq r_{s} \leq 0.91\right)$ between the NTS categories 1-6 related to the assessment of NTSs (table 1, question category I7). Correlation between these six categories and the skill category 'coping with fatigue' was distinctly smaller $\left(0.62 \leq \mathrm{r}_{\mathrm{s}} \leq 0.76\right)$.

Visual inspection of the bar charts of the frequency of simulation-based training (figure 2) and assessment (figure 3) indicate that HCMs generally appear to train and undergo assessment more frequently, and physicians less frequently, than pilots.

These apparent differences in simulation-based training between groups of crew members are, however, not statistically significant (table 2, question category I6). The tendency for ORs to be larger for HCMs and smaller for physicians can be seen across all skill categories, but CIs are wide.

Physicians are assessed significantly less frequently than pilots (table 2, question category I7), but the differences between HCMs and pilots are not statistically significant. There is, however, also a tendency here for ORs to be larger for HCMs and smaller for physicians across all skill categories.

Compared with employees working for the flight operator (pilots and HCMs), employees working for the health enterprise (physicians) undergo statistically significantly less frequent simulation-based training (table 3, question category I6) and assessment (table 3, question category I7).

All professional groups work longer hours and are exposed to significant fatigue. Of the hospital employees, 50 out of 82 
Table 1 Inter-item correlations (Spearman $r, r_{s}$ ) between each of the seven generic non-technical skills (NTSs) in the question categories 16 and I7

\begin{tabular}{|c|c|c|c|c|c|c|c|c|}
\hline Question category & NTS category & 1 & 2 & 3 & 4 & 5 & 6 & 7 \\
\hline 16: Simulation-based training & 1. Decision-making & - & & & & & & \\
\hline \multirow[t]{6}{*}{ of NTSs $(144 \leq n \leq 150)$} & 2. Leadership & 0.85 & - & & & & & \\
\hline & 3. Communication & 0.89 & 0.88 & - & & & & \\
\hline & 4. Situation awareness & 0.81 & 0.80 & 0.85 & - & & & \\
\hline & 5. Teamwork & 0.85 & 0.77 & 0.85 & 0.83 & - & & \\
\hline & 6. Managing stress & 0.77 & 0.71 & 0.71 & 0.74 & 0.68 & - & \\
\hline & 7. Coping with fatigue & 0.59 & 0.60 & 0.56 & 0.61 & 0.53 & 0.78 & - \\
\hline \multirow[t]{7}{*}{ 17: Assessment of NTSs $(145 \leq n \leq 149)$} & 1. Decision-making & - & & & & & & \\
\hline & 2. Leadership & 0.91 & - & & & & & \\
\hline & 3. Communication & 0.90 & 0.89 & - & & & & \\
\hline & 4. Situation awareness & 0.86 & 0.81 & 0.90 & - & & & \\
\hline & 5. Teamwork & 0.88 & 0.87 & 0.83 & 0.82 & - & & \\
\hline & 6. Managing stress & 0.80 & 0.80 & 0.77 & 0.80 & 0.82 & - & \\
\hline & 7. Coping with fatigue & 0.65 & 0.66 & 0.62 & 0.68 & 0.62 & 0.76 & - \\
\hline
\end{tabular}

(61\%) were on call for more than 72 consecutive hours on a regular basis. Of these, $79 \%$ did not have any training in coping with fatigue. In contrast, 72 out of 73 (99\%) pilots and HCMs were on call for more than 3 days in a row. Of these, $54 \%$ did not have any training in coping with fatigue.

\section{DISCUSSION}

This is the first study of simulation-based training and assessment of NTSs in the Norwegian HEMS. We found considerable variation in the extent of simulation-based training and assessment of NTSs among the crew members. A significant number of crew members reported complete absence of simulationbased training and assessment.

The strength of correlations between the NTS categories was generally high. That is, the more respondents train or undergo assessment in one of the NTS categories, the more they generally train or undergo assessment in other NTS categories. The item 'coping with fatigue' differs from the other skill categories, which might reflect the fact that it is not an explicit skill category but rather an item that influences the others.

\section{Lack of simulation-based training}

The need for training in complex environments is often underestimated. ${ }^{4}$ Our data indicate that, compared with HCMs and pilots, a statistically significantly smaller proportion of HEMS physicians have undergone simulation-based NTS training. Similarly, as early as 2001, it was suggested that anaesthesiologists lacked training in NTSs for critical situations in hospitals. ${ }^{10}$ To overcome this, Gaba and colleagues created a simulationbased curriculum based on key principles from aviation CRM training. ${ }^{10}$ Differences in task environment and professional cultures may help to provide an answer to what we have revealed.
(1) decision making

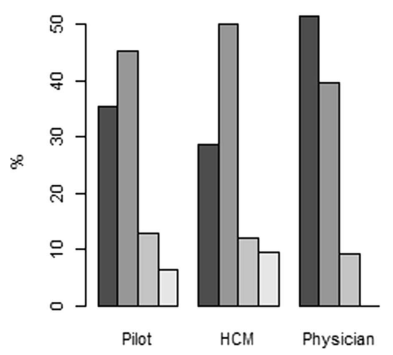

(5) teamwork

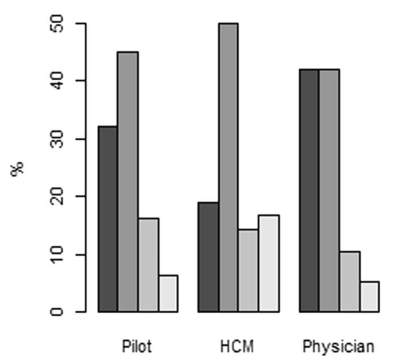

(2) leadership

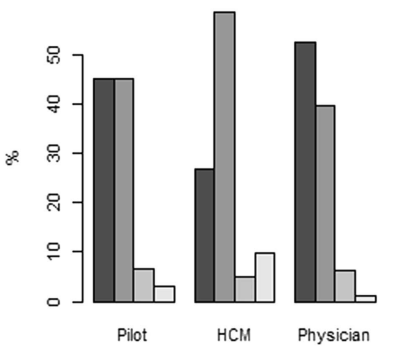

(6) managing stress

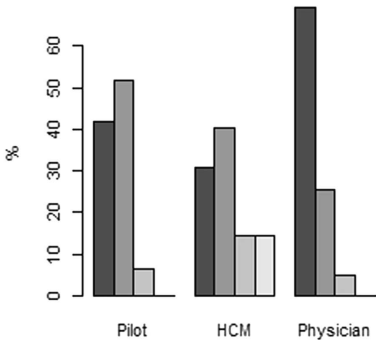

(3) communication

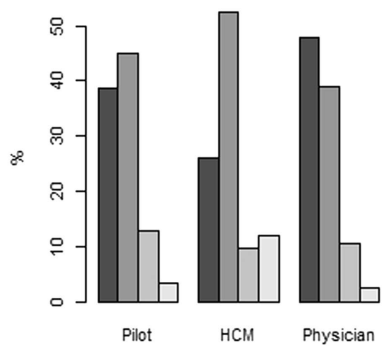

(7) coping with fatigue

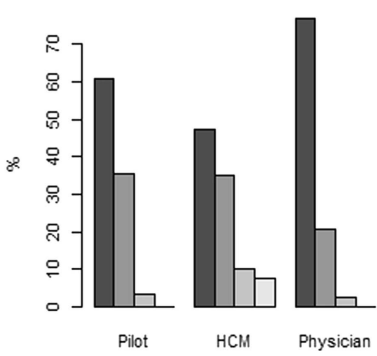

Figure 2 Multidisciplinary, prehospital simulation-based training of generic non-technical skills (1-7) in 2011. ${ }^{1}$ Complete answers from each of the three professional groups in a HEMS crew (horizontal axis) across four ordinal categories of frequency within a year (box). Proportion of individuals (relative frequency, \%) within each professional group on vertical axis. HEMS, helicopter emergency medical service. 
(1) decision making

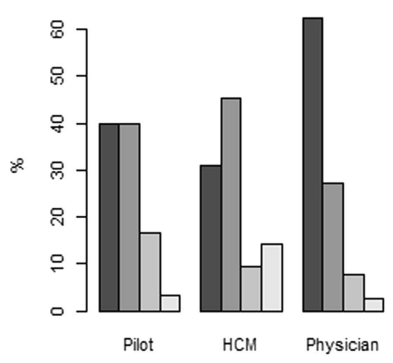

(5) teamwork

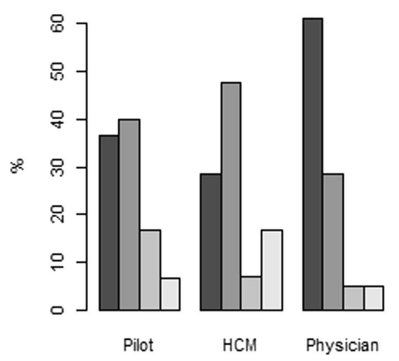

(2) leadership

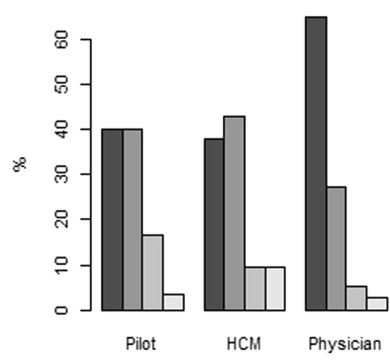

(3) communication

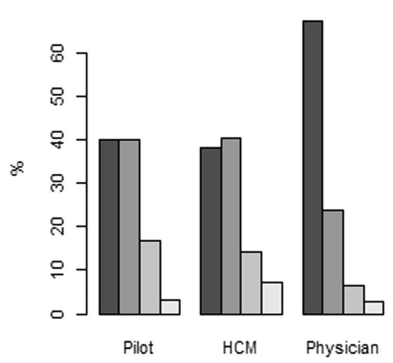

(4) situation awareness

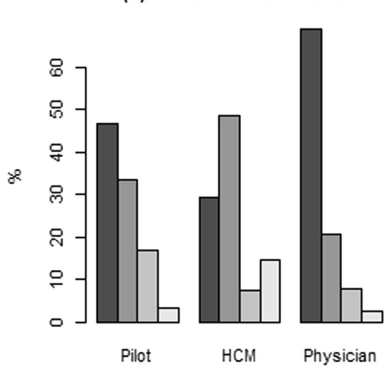

Figure 3 Assessment of seven (1-7) generic non-technical skills in $2011 .{ }^{1}$ Complete answers from each of the three professional groups in a HEMS crew (horizontal axis) across four ordinal categories of frequency within a year (box). Proportion of individuals (relative frequency, \%) within each professional group on vertical axis. HEMS, helicopter emergency medical service.

It is claimed that aviation is more procedure-based than prehospital critical care, and hence it is easier to train and assess crew in its process. Airline staff also have longer traditions of recurrent training in, and evaluation of, NTSs than medical staff. ${ }^{10}$ In addition, the professional cultures differ markedly. Aviation staff have managed to change the professional culture into one that recognises human limitations and the need for NTS training, ${ }^{20}$ while cultural resistance against extending CRM training into the medical domain has been reported. ${ }^{21}$ Another obstacle to training is that simulation-based training is a time-consuming and often costly activity that will disrupt clinical duties. A competent facilitator is needed to design and prepare a scenario, and the crew members need time for training and debriefing.

\section{Lack of assessment}

Similar to our findings on simulation-based training, physicians undergo NTS assessment significantly less often than the other professional groups. Domain-specific NTSs have been identified, and assessment tools have been developed, for teams and individuals in medical teams, but not in the context of prehospital critical care ${ }^{22}$ and HEMS. Without a frame of reference, the description and evaluation of NTSs will be ambiguous. What is assessed, how it is assessed and how this information is used will vary - and ultimately training may not be assessed at all. This may well be the reason for the lack of assessment in our data. Without carrying out thorough evaluations, it can be difficult to test skills, to provide feedback on skill development, to

Table 2 OR with 95\% Cls for physicians and HEMS crew members (HCMs) having undergone simulation-based training (question category 16) and assessment (question category 17) of seven (1-7) generic non-technical skills (NTSs), ${ }^{1}$ compared with the group of pilots

\begin{tabular}{|c|c|c|c|c|c|c|c|}
\hline \multirow[b]{2}{*}{ Question category } & \multirow[b]{2}{*}{ NTS category (n) } & \multicolumn{3}{|l|}{ Physician } & \multicolumn{3}{|l|}{$\mathrm{HCM}$} \\
\hline & & OR $(95 \% \mathrm{Cl})$ & p Value & $\mathrm{n}_{\text {phys }}$ (missing) & OR $(95 \% \mathrm{Cl})$ & p Value & $\mathrm{n}_{\mathrm{HCM}}$ (missing) \\
\hline \multirow{7}{*}{$\begin{array}{l}\text { 16: Simulation-based } \\
\text { training of NTSs }\end{array}$} & 1. Decision-making $(n=149)$ & $0.52(0.22$ to 1.24$)$ & 0.139 & $76(6)$ & $1.38(0.51$ to 3.72$)$ & 0.530 & $42(0)$ \\
\hline & 2. Leadership $(n=150)$ & $0.74(0.32$ to 1.71$)$ & 0.486 & $78(4)$ & 2.25 (0.84 to 6.04$)$ & 0.109 & $41(1)$ \\
\hline & 3. Communication $(n=150)$ & $0.68(0.29$ to 1.60$)$ & 0.379 & $77(5)$ & 1.78 (0.66 to 4.83$)$ & 0.257 & $42(0)$ \\
\hline & 4. Situation awareness $(n=150)$ & 0.67 (0.29 to 1.55$)$ & 0.348 & $77(5)$ & $2.04(0.76$ to 5.48$)$ & 0.160 & $42(0)$ \\
\hline & 5. Teamwork $(n=149)$ & $0.66(0.27$ to 1.58$)$ & 0.346 & $76(6)$ & 2.02 (0.69 to 5.94$)$ & 0.200 & $42(0)$ \\
\hline & 6. Managing stress $(n=151)$ & 0.32 (0.14 to 0.76$)$ & 0.010 & $78(4)$ & 1.61 (0.61 to 4.24$)$ & 0.334 & $42(0)$ \\
\hline & 7. Coping with fatigue $(n=146)$ & $0.46(0.18$ to 1.17$)$ & 0.103 & $78(4)$ & $1.71(0.64$ to 4.55$)$ & 0.284 & $40(2)$ \\
\hline \multirow[t]{7}{*}{ 17: Assessment of NTSs } & 1. Decision-making $(n=149)$ & $0.40(0.17$ to 0.96$)$ & 0.039 & $77(5)$ & 1.49 (0.56 to 3.97$)$ & 0.428 & $42(0)$ \\
\hline & 2. Leadership $(n=149)$ & $0.36(0.15$ to 0.86$)$ & 0.021 & $77(5)$ & $1.08(0.42$ to 2.83$)$ & 0.870 & $42(0)$ \\
\hline & 3. Communication $(n=148)$ & 0.33 (0.14 to 0.78$)$ & 0.012 & $76(6)$ & $1.08(0.42$ to 2.83$)$ & 0.870 & $42(0)$ \\
\hline & 4. Situation awareness $(n=148)$ & $0.40(0.17$ to 0.94$)$ & 0.036 & $77(5)$ & $2.12(0.79$ to 5.65$)$ & 0.136 & $41(1)$ \\
\hline & 5. Teamwork $(n=149)$ & 0.37 (0.15 to 0.88$)$ & 0.025 & $77(5)$ & 1.45 (0.58 to 3.93$)$ & 0.469 & $42(0)$ \\
\hline & 6. Managing stress $(n=149)$ & $0.29(0.12$ to 0.69$)$ & 0.005 & $77(5)$ & $1.24(0.48$ to 3.23$)$ & 0.655 & $42(0)$ \\
\hline & 7. Coping with fatigue $(n=146)$ & $0.38(0.15$ to 0.98$)$ & 0.046 & $77(5)$ & 1.64 (0.64 to 4.34$)$ & 0.318 & $39(3)$ \\
\hline
\end{tabular}


Table 3 Proportion (\%) of crew members in helicopter emergency medical services (HEMS) working for the health enterprise (physicians) and for the flight operator (HEMS crew members (HCMs) and pilots) who have undergone simulation-based training (question category I6) and assessment (question category 17) of seven (1-7) generic non-technical skills (NTSs) ${ }^{1}$

\begin{tabular}{|c|c|c|c|c|c|}
\hline Question category & NTS category & Health enterprise employee & Flight operator employee & $\mathrm{N}$ (missing) & p Value (2-sided) \\
\hline \multirow[t]{7}{*}{ 16: Simulation-based training of NTSs } & 1. Decision-making & $37 / 76(48.7 \%)$ & $50 / 73(68.5 \%)$ & $149(6)$ & 0.020 \\
\hline & 2. Leadership & $37 / 78(47.4 \%)$ & $47 / 72(65.3 \%)$ & $150(5)$ & 0.033 \\
\hline & 3. Communication & $40 / 77(51.9 \%)$ & $50 / 73(68.5 \%)$ & $150(5)$ & 0.046 \\
\hline & 4. Situation awareness & $37 / 77(48.1 \%)$ & $49 / 73(67.1 \%)$ & $150(5)$ & 0.021 \\
\hline & 5. Teamwork & $44 / 76(57.9 \%)$ & $55 / 73(75.3 \%)$ & $149(6)$ & 0.037 \\
\hline & 6. Managing stress & $24 / 78(30.8 \%)$ & $47 / 73(64.3 \%)$ & $151(4)$ & $<0.001$ \\
\hline & 7. Coping with fatigue & $18 / 78(23.8 \%)$ & $32 / 68(47.1 \%)$ & $146(9)$ & 0.003 \\
\hline \multirow[t]{7}{*}{ 17: Assessment of NTSs } & 1. Decision-making & $29 / 77(37.7 \%)$ & $47 / 72(65.3 \%)$ & $149(6)$ & 0.001 \\
\hline & 2. Leadership & $27 / 77(35.1 \%)$ & $44 / 72(61.1 \%)$ & $149(6)$ & 0.002 \\
\hline & 3. Communication & $25 / 76(32.9 \%)$ & $44 / 72(61.1 \%)$ & $148(7)$ & 0.001 \\
\hline & 4. Situation awareness & $24 / 77(31.2 \%)$ & $45 / 71(63.3 \%)$ & $148(7)$ & $<0.001$ \\
\hline & 5. Teamwork & $30 / 77(38.9 \%)$ & $49 / 72(68.1 \%)$ & $149(6)$ & $<0.001$ \\
\hline & 6. Managing stress & $21 / 77(27.3 \%)$ & $43 / 72(59.7 \%)$ & $149(6)$ & $<0.001$ \\
\hline & 7. Coping with fatigue & $14 / 77(18.2 \%)$ & $30 / 69(43.5 \%)$ & $146(9)$ & 0.001 \\
\hline
\end{tabular}

Comparison of health enterprise employees with flight operator employees using Fisher's exact test (two-sided). Significance at level 0.05 .

point out strengths and identify training needs, and to determine whether an NTS training programme (CRM) is effective at improving the skills in question. ${ }^{12}$

\section{Teamwork}

Single- and multi-disciplinary team training are complementary methods, and personnel should participate in both to develop teamwork skills. ${ }^{10}$ Our data imply that not all simulation training takes place within the framework of a complete multidisciplinary HEMS crew.

Team performance may directly affect patient safety. ${ }^{10} \quad 16$ A shared understanding - a shared mental model—of the task in hand and of the other team members' roles has been identified as one important characteristic of a high-performance team. ${ }^{10} 12$ The physician and the HCM are primarily responsible for providing patient care on-scene. The pilot is the only crew member with no formal medical skill competencies, and he is thus least qualified to take part in the medical treatment. However, the pilot is often involved in simpler patient-related tasks to assist the medical crew, such as checking of medical equipment, resuscitation and preparing the patient for transport on the stretcher. This is similar to the physician, who does not have formal flight training, but has responsibilities related to flight safety both during take-off and landing and in-flight in order to supplement the pilot and the HCM. These tasks require teamwork and understanding across disciplines.

\section{Duty hours}

Regularly scheduled on-call duty for Norwegian HCMs lasts for up to 7 consecutive days around the clock. This is much longer than similar rotor-wing air medical programmes in the USA, where the maximum shift length has been reported to be $48 \mathrm{~h}^{23}$ A high number of duty hours a week is common among emergency medical service providers, and has been suggested to be in part culturally determined. Long shifts and on-call working is recognised as a risk to patients and operational safety. $^{23} 24$ The workload and frequency of HEMS missions will vary during the on-call period. On-duty rest and sleep is permitted for all crew members in Norway and must be obtained between missions. Working at night, for irregular hours, is inevitable and results in disrupted sleep and a displaced sleep schedule, which might affect mental performance, health and the risk of adverse events. $^{23} 24$ To prevent fatigue, pilots and HCMs are protected by flight time limitations and rest time rules, ${ }^{25}$ but these regulations do not deal with quality of rest and sleep between missions. HEMS physicians in Norway are protected by the same rest and sleep regulations as pilots and HCMs, but the regulations are enforced differently in different HEMS bases. Crew members in our study reported that they receive limited training on how to recognise and cope with fatigue.

\section{Strengths and limitations of the study}

The response rate for the survey was $81.8 \%$, with few data missing, which is considered satisfactory and is a strength of the study. The study is limited by its small sample size. Despite a high response rate, which increases effective sample size and reduces non-responder bias, the number of respondents was too small to detect statistically significant differences between all the professional groups.

The study was limited to a set of seven broad generic NTS categories claimed to have general applicability across a wide range of high-risk work settings. ${ }^{12}$ The questions did not differentiate between composite team assessment and individual performance assessment. ${ }^{12}$

We did not include conceptual explanations and definitions in our questionnaire. We assumed that the crew members already shared a common vocabulary for discussing the basic principles of NTSs, since CRM training is mandatory for all crew members in Norway.

In order to achieve maximum response rates, both a paper version and an electronic version of the questionnaire were made available at the same time in the data-collection period. We emphasised that each respondent had to fill out only one form each, either paper or electronic. We considered the likelihood of multiple responses from one individual very small and the advantage of a high response rate correspondingly large. There is still a possibility that a single respondent may have filled out more than one form.

The questionnaire was anonymous, and responding to the questionnaire was voluntary. However, there is a possibility that respondents to this type of questionnaire do not respond truthfully, or do not remember details exactly. This may result in under- or over-reporting. We do not have any information about the non-responders. 


\section{Implications}

This study has implications for current practice and future research. Existing training requirements, and assessment criteria, for Norwegian HCMs are based on generalised statements of performance outputs. They do not clearly specify how often training for and assessment of NTSs should be. Mandatory NTS training requirements for crew members in the civilian Norwegian HEMS need to be specified as an incentive to train, with a view to licensing and registration. Special emphasis needs to be placed on patient safety issues relating to fatigue and sleep homoeostasis among crew members in HEMS. Future research might explore how to increase frequency of simulation-based NTS training with minimal disruption to clinical duties and with little expense.

\author{
Author affiliations \\ ${ }^{1}$ Department of Research and Development, Norwegian Air Ambulance Foundation, \\ Drøbak, Norway \\ ${ }^{2}$ Department of Global Public Health and Primary Care, University of Bergen, \\ Bergen, Norway \\ ${ }^{3}$ National Centre for Emergency Primary Health Care, Uni Research Health, Uni \\ Research, Bergen, Norway \\ ${ }^{4}$ Department of Industrial Economics, Risk Management and Planning, University of \\ Stavanger, Stavanger, Norway \\ ${ }^{5}$ Department of Health Sciences, University of Stavanger, Stavanger, Norway \\ ${ }^{6}$ Air Ambulance Department, Oslo University Hospital, Oslo, Norway \\ ${ }^{7}$ Faculty of Health and Occupational Studies, University of Gävle, Gävle, Sweden \\ ${ }^{8}$ Department of Biostatistics, Institute of Basic Medical Sciences, University of Oslo, \\ Oslo, Norway
}

Acknowledgements We would particularly like to thank all pilots, HCMs, nurses and physicians in the Norwegian civilian HEMS for their time and willingness to participate in this survey. Ingvild Dalen, statistician at the Stavanger University Hospital, contributed with ideas for statistical calculations to an early version of this paper. Finally, we thank all members of the Norwegian Air Ambulance Foundation for the financial support that made this research possible.

Contributors HBA: guarantor of the manuscript and responsible for the integrity of the data and the accuracy of the data analysis; conceived and designed the study; data collection, statistical analysis and interpretation of the data; and drafting, writing, review and incorporating coauthor feedback, revision, and final approval of the submission. SJMS: contributed to the concept and design, analysis and interpretation of the data, and the writing, review, revision and approval of the manuscript. LSÖ: contributed to the concept and design; analysis and interpretation of the data; and the drafting, writing, review, revision and approval of the manuscript. JR: contributed to statistical analysis and interpretation of the data; and the writing, review, revision of the article for important intellectual content, and approval of the manuscript. GTB: contributed to the concept and design; analysis and interpretation of the data; and the drafting, writing, review, revision and approval of the manuscript.

Funding This research was fully funded by the Norwegian Air Ambulance Foundation.

Competing interests HBA is employed in a $70 \%$ clinical position at Stavanger University Hospital where he works as a consultant doctor at the Department of Anaesthesiology and Intensive Care. He is working in the prehospital services at the air ambulance base in Stavanger as part of his clinical position. HBA is a PhD fellow in a $50 \%$ position at the Norwegian Air Ambulance Foundation and the University of Bergen.

Ethics approval The regional ethics committee of South-Eastern Norway (reference number 2010/3326) and the Norwegian Social Science Data Services reviewed and approved the study.

Provenance and peer review Not commissioned; externally peer reviewed.

Data sharing statement There exist additional data on safety climate and crew members' perceptions of simulation-based training and assessment. These data are available to HBA, GTB, SJMS and LSÖ.
Open Access This is an Open Access article distributed in accordance with the Creative Commons Attribution Non Commercial (CC BY-NC 4.0) license, which permits others to distribute, remix, adapt, build upon this work non-commercially, and license their derivative works on different terms, provided the original work is properly cited and the use is non-commercial. See: http://creativecommons.org/ licenses/by-nc/4.0/

\section{REFERENCES}

1 Grote G. Safety management in different high-risk domains - All the same? Saf Sci 2012;50:1983-92.

2 Seymour CW, Kahn JM, Schwab CW, et al. Adverse events during rotary-wing transport of mechanically ventilated patients: a retrospective cohort study. Crit Care 2008; 12:R71.

3 Patterson PD, Lave JR, Martin-Gill C, et al. Measuring adverse events in helicopter emergency medical services: establishing content validity. Prehosp Emerg Care 2014; 18:35-45.

4 Gordon M, Darbyshire D, Baker P. Non-technical skills training to enhance patient safety: a systematic review. Med Educ 2012;46:1042-54.

5 Flin R, Patey R. Improving patient safety through training in non-technical skills. Br Med J 2009;339:b3595.

6 Helseforetakenes nasjonale Luftambulansetjeneste ANS. Årsrapport 2013 (Annual report air ambulance services 2013, Norway). http://www.luftambulanse.no/ årsrapport-2013-er-klar (accessed 13 Sep 2014).

7 Dalto JD, Weir C, Thomas F. Analyzing communication errors in an air medical transport service. Air Med J 2013;32:129-37.

8 Geogiou A, Lockey DJ. The performance and assessment of hospital trauma teams. Scand J Trauma Resusc Emerg Med 2010;18:66.

9 Singh JM, MacDonald RD, Bronskill SE, et al. Incidence and predictors of critical events during urgent air-medical transport. CMAJ 2009;181:579-84.

10 Gaba D, Howard SK, Fish KJ, et al. Simulation based training in anesthesia crisis resource management (ACRM): a decade of experience. Simulation Gaming 2001;32:175-93.

11 O'Connor P, Campbell J, Newon J, et al. Crew resource management training effectiveness: a meta-analysis and some critical needs. Int J Aviat Psychol 2008;18:353-68.

12 Flin R, O'Connor P, Crichton M. Safety at the Sharp End. A guide to non-technical skills. Aldershot: Ashgate, 2008.

13 Kohn LT, Corrigan J, Donaldson M. To err is human: building a safer health system. Washington, DC: National Academies Press (US), 2000.

14 Wahr JA, Prager RL, Abernathy JH III, et al. Patient safety in the cardiac operating room: human factors and teamwork: a scientific statement from the American Heart Association. Circulation 2013;128:1139-69.

15 Gjeraa K, Møller TP, Østergaard D. Efficacy of simulation-based trauma team training of non-technical skills. A systematic review. Acta Anaesthesiol Scand 2014;58:775-87.

16 Manser T. Teamwork and patient safety in dynamic domains of healthcare: a review of the literature. Acta Anaesthesiol Scand 2009;53:143-51.

17 Lorello GR, Cook DA, Johnson RL, et al. Simulation-based training in anaesthesiology: a systematic review and meta-analysis. Br J Anaest 2014;112:231-45.

18 Abrahamsen HB, Abrahamsen EB, Lossius HM. Safety culture in the air ambulance services. Tidsskr Nor Legeforen 2012;132:797-8.

19 Flin R, Maran N. Identifying and training non-technical skills for teams in acute medicine. Qual Saf Health Care 2004;13:i80-4.

20 Sexton B, Thomas EJ, Helmreich RL. Error, stress and teamwork in medicine and aviation: cross sectional surveys. BMJ 2000;320:745-9.

21 Timmons $S$, Baxendale B, Buttery $A$, et al. Implementing human factors in clinical practice. Emerg Med J 2015;32:368-72.

22 Shields A, Flin R. Paramedics' non-technical skills: a literature review. Emerg Med J 2013;30:350-4.

23 Frakes MA, Kelly JG. Shift length and on-duty rest patterns in rotor-wing air medical programs. Air Med J 2004;23:34-9.

24 Åkerstedt T. Altered sleep/wake patterns and mental performance. Physiol Behav 2007;90:209-18.

25 EU-OPS Subpart Q: Flight and duty time limitations and rest requirements. Official Journal of the European Union L254/223-L254/230. In: Commission regulation (EC) No 859/2008 of August 2008 amending Council Regulation (ECC) No 3922/91 as regards common technical requirements and administrative procedures applicable to commercial transportation by aeroplane. http://www.luftfartstilsynet.no/regelverk/ Europeisk_regelverk/article1325.ece (accessed 30 Apr 2014). 